\title{
Paediatric Germ Cell Tumours: Case Study Oncology Centre of Basra Between 2004-2015
}

\author{
Nayef J. Farhan Al-Khafaji ${ }^{1}$, Wasan H. Saud Al-Ibadi ${ }^{2}$, Aqila A. Sahi Kadhim ${ }^{3}$ \\ \{nayef_farhan@yahoo.com ${ }^{1}$,wasanhameed27@yahoo.com ${ }^{2}$, akilaabood@yahoo.com ${ }^{3}$ \} \\ Basra Special Children Hospital, Basra, Iraq ${ }^{1,2}$ \\ Gynecologist in Basra general hospital ${ }^{3}$
}

\begin{abstract}
The incidence of germ cell tumours (GCT) was 2.9\% in all malignant tumours of children less than 16 years old with the occurrence of GCT in male to female ratio was 1.0:1.1. The main aim of this study is to exhibit the outcome and treatment of children less than 16 years old in Basra Oncology Centre in Iraq between 2004-2015. In the oncology Centre of Basra 45 cases of GCT age 1 to 156 months (mean 78.5 months). The sample divide as a following, female $24(46.7 \%)$ and male $21(53.3 \%)$ with gonadal $21(46.7 \%)$ testes $9(20 \%)$ and ovary $12(26.7 \%)$. Histologically, in our data, the most common GCT was teratomas 2 mature teratomas these two cases were excluded from the current study, 20 cases immature $44.4 \%$, followed by yolk sac tumours nineteen cases $(\mathrm{n}: 19=42.2 \%)$, dysgerminoma (n: $4=8.9 \%$ ) and embryonal carcinoma (n: $2=4.4 \%$ ). Based on the results, about 5 years overall survival (OS) is $88.9 \%$. During the previous years, the surgical, chemotherapy, and radiotherapy guidelines for (GCT) have been purified to decrease the early and late morbidities and associated with improving survival. Used of Platinum (carboplatin /cisplatin) the outcome of children with (GCT) has improved 5-year survival.
\end{abstract}

Keywords: Germ cell tumours, Platinum, children, Basra

\section{Introduction}

Germ cell tumour (GCT) incidence is approximately 2-3\% of paediatric malignancies. The prevalence of Germ Cell Tumours in white children is 2.5 per million in and in African American children is 3.0 per million under 15 years of age. comparison the incidence of GCT in male: female the ratio was 1.0:1.1[1].

GCTs are highly occurrence in the gonadal (ovaries and testes) follow by extragonadal sites like mediastinum, pineal and sacrococcygeal regions, and retroperitoneum $[1,2]$. The germ cells are the source and origin of sperm and ova and after fertilization then produce all the embryonic and extraembryonic structures of the developing embryo. totipotential germ cell is the origin of Yolk sac tumours which is differentiated to extraembryonic structures while Teratomas are origin from the second type of cell which produces the embryonic tissues so that neoplasms contain tissues from all three germ layers (ectoderm, endoderm, and mesoderm) so Teratomas are either begin or malignant depending on present or absent of malignant germ cell elements (embryonal carcinomas, germinomas, endodermal sinus tumours or choriocarcinomas) or primitive neuroectodermal tumours which is a rare malignant somatic element in paediatric[1, $3]$. 
GCTs show numerous histologic subtypes. The features of each histologic subtype are independent of presenting clinical characteristics; tumour biology depends on the site of origin and the clinical behaviour vary with state, site of origin, and age of the patient. The introduction of platinum-based chemotherapy(carboplatin and cisplatin) increased the overall survival by more than $80 \%$, even in late stages $[2,4]$.

This project aimed to display our oncological centre experience with the epidemiological and clinical characteristics, handling, management, and outcome in germ cell tumours (GCTs) over 12 years.

\section{Materials and Methods}

Forty-five children with GCT were admitted to our oncological centre between 2004 and 2015. The pause date for analysis of these patients was February 2016 and only follow members in our study for five years. Their pathologic features, clinical characteristics, methods of treatment, relapse, and survival outcomes were retrospectively evaluated. The tumours were stratified according to the Dehner system[5]. In our study, two mature teratoma patients were diagnosed and excluded. Table 1 shows the Children's Oncology Group (COG) classification system for staging was used[5, 6].

Table 1. Children's Oncology Group (COG) staging system.

\begin{tabular}{c|l}
\hline \hline Stage I & $\begin{array}{l}\text { Complete surgical removal associated with margins tumour-free. } \\
\text { Microscopic residual tumour or persistently raised tumour markers, } \\
\text { with negative lymph nodes. }\end{array}$ \\
Stage II & $\begin{array}{l}\text { Biopsy only, or gross residual disease, lymph node involvement. } \\
\text { Metastatic tumour. }\end{array}$ \\
Stage III
\end{tabular}

The quantitative variables were described using median and range; qualitative variables were described using frequency and percentages. We used the chi-square test to examine the relationship between pairs of variables. Overall survival (OS) and relapse-free survival (RFS) distributions were estimated by the method of Kaplan-Meier survival analyses. Using the Cox proportional hazards regression model, multivariate analyses were performed to determine the variables that were independently predictive of prognosis.

\section{Results}

Throughout twelve years, forty-five patients with GCTs were admitted to the Oncology Centre of Basra. Distribution data of the 45 patients according to the sex 24 were female and 21 were male, as illustrated in Table 2.

Table 2. Sex incidence in GCT patients in our study. 


\begin{tabular}{ccc}
\hline Female & 24 & 53.3 \\
Male & 21 & 46.6 \\
\hline
\end{tabular}

Their median age was 78.5 months (1-156 months). The children society classified according to the age to the following group[7]:

- Neonates: birth up to 1 month.

- Infants: 1 up to 24 months; (1months to 2years)

- Children: 24 up to 144 months; (2 years to12 years)

- Adolescents: 144 up to 192 months;(12 years to 16 years) [7]

Table 3. Age distribution in GCT and percentage.

\begin{tabular}{lcc}
\hline \multicolumn{1}{c}{ Age } & NO. & percentage \\
\hline birth up to 1 month & 1 & 2.2 \\
1 up to 24 months & 14 & 31.1 \\
24 up to 144 months & 29 & 64.4 \\
144 up to 192 months & 1 & 2.2 \\
\hline
\end{tabular}

As shown in Table 3, the most common age associated with GCT were 24 up to144 months of age (64.4\%). The sex distribution in GCT in Oncology Centre of Basra was female more than male; the female was $24(53.3 \%)$ and the male was $21(46.6 \%)$; male; female (1:1.1). in all age groups either same or female more than male. The abdominal or pelvic mass associated with abdominal distension was present in 20 patients(43.5\%) which is identical to other signs included sacrococcygeal mass in 20 patients $(43.5 \%)$. Abdominal pain was the second most frequent symptom, reported in 19 patients $(41.3 \%)$ either acute, chronic, or both. The third important symptom was increased testicular size with a palpable mass in 10 patients $(21.7 \%)$. Other symptoms and signs included constipation, precocious puberty, urinary symptoms, nausea, vomiting.

Table 4. Occurrence of signs and symptoms in GCT.

\begin{tabular}{ccc}
\hline Symptoms and Signs & No. & \% \\
\hline Abdominal distension & 20 & 43.5 \\
Sacrococcygeal mass & 20 & 43.5 \\
Abdominal pain & 19 & 41.3 \\
Increase testicular size & 10 & 21.7 \\
\hline
\end{tabular}

The abdomen ultrasound and Computed tomography which were the most common investigation used diagnosis and follow-up of patients with GCT. abdominal ultrasound, which was the most common investigation, used in all patients. Computed tomography was performed for most patients. Magnetic resonance imaging was not performed. Serum alpha-fetoprotein (AFP) level:
1) Normal
$(<20 \mathrm{IU} / \mathrm{ml})$
2) Moderate
$(20-399 \mathrm{IU} / \mathrm{ml})$
3) Elevated
$(\geq 400 \mathrm{IU} / \mathrm{ml})$ 
Alpha-fetoprotein (AFP) was measure in 27 (60\%) patients in the oncology Centre of Basra. four cases were normal $<20 \mathrm{IU} / \mathrm{ml}(8.9 \%)$, thirteen cases Moderate 20-399IU/ml $(28.8 \%)$ and ten cases Elevated $\geq 400 \mathrm{IU} / \mathrm{ml}(22.2 \%)$. AFP measurement according to the tumour histopathology Teratoma 14 patients $(31.1 \%)$, yolk sac 12 patients $(26.6 \%)$, and embryonal carcinoma one case (2\%). Unfortunately, eighteen cases (40\%) with GCT did not measure the tumour markers because of socio-economic difficulties.

Table 5. Alpha fetoprotein level evaluation in GCT.

\begin{tabular}{ccc}
\hline AFP level & No. & \% \\
\hline Not measure & 18 & 40 \\
Normal $(<20 \mathrm{IU} / \mathrm{ml})$ & 4 & 8.9 \\
Moderate $(20-399 \mathrm{IU} / \mathrm{ml})$ & 13 & 28.8 \\
Elevated $(\geq 400 \mathrm{IU} / \mathrm{ml})$ & 10 & 22.2 \\
\hline
\end{tabular}

The most common site for the presence of GCT was the extragonadal site 24 patients (53.3\%) extragonadal (4 retroperitoneum, 20 sacrococcygeal) as presented in Table 5, the second place was ovaries 12 patients (26.6\%) and the testes were lesser site 9 patients $(20.1 \%)$.

Table 6. GCT site distribution.

\begin{tabular}{ccc}
\hline Location & No. & \% \\
\hline extragonadal & 24 & 53.3 \\
ovaries & 12 & 26.6 \\
testes & 9 & 20.1 \\
\hline
\end{tabular}

The testicular tumour mainly occurs at age 1 month to 2 years( 5 cases $11.1 \%)$, the ovarian tumours most common in 2 to 12 years( 11 cases $24.4 \%$ ), and also the extragonadal tumour commonly in 2-12 years old (14 cases $31.1 \%$ ). Histologically, teratomas were found most frequently ( 2 mature, 20immature $44.4 \%$ ), followed by yolk sac tumours (n:19=42.2\%), dysgerminoma (n:4=8.9\%), and embryonal carcinoma (n: $2=4.4 \%$ ), as illustrated in Table 7 .

Table 7. GCT histological occurrence.

\begin{tabular}{ccc}
\hline Histology & No. & \% \\
\hline teratomas & 20 & 44.4 \\
yolk sac tumours & 19 & 42.2 \\
dysgerminoma & 4 & 8.9 \\
embryonal carcinoma & 2 & 4.4 \\
\hline
\end{tabular}

The distribution of GCT according to the age; teratomas are most frequently in 2-12 years old patients and yolk sac tumours occur mostly in less than 2 years old patients, as shown in Fig 1. 


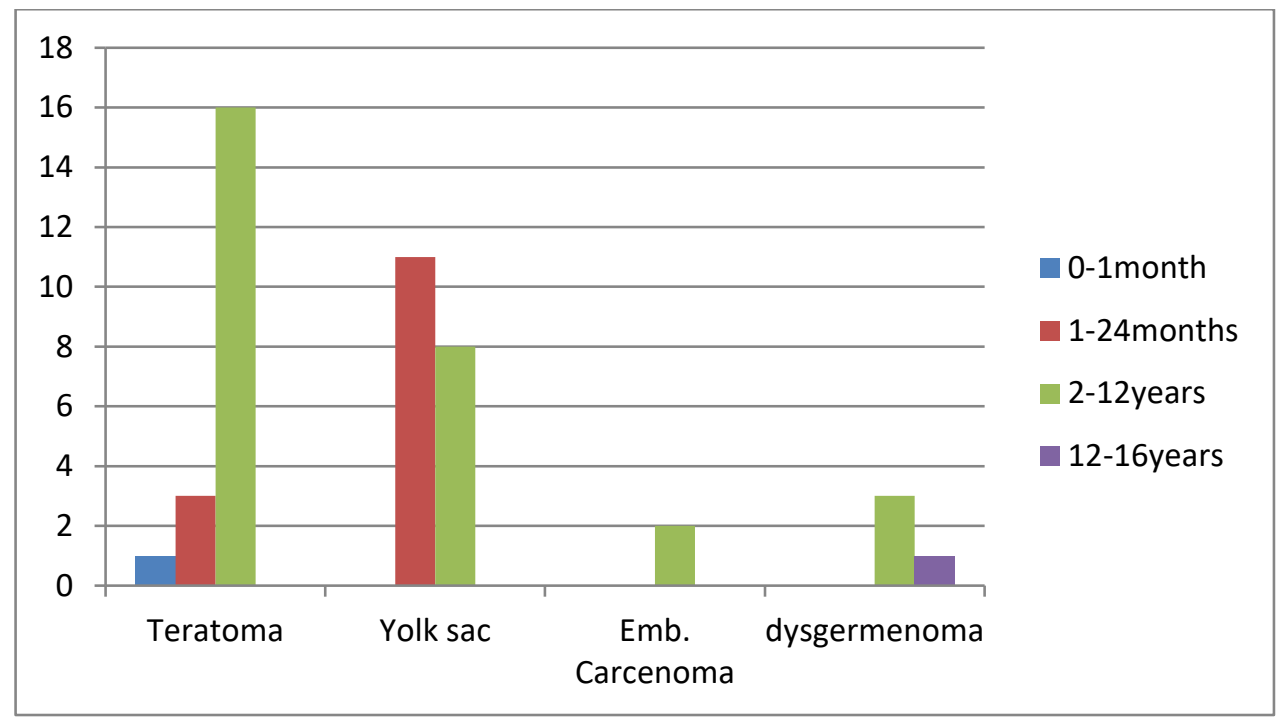

Fig 1. GCT histological distribution according to the age.

In the oncology Centre of Basra found teratomas most frequently occur in females while the yolk sac occurs mostly in males.

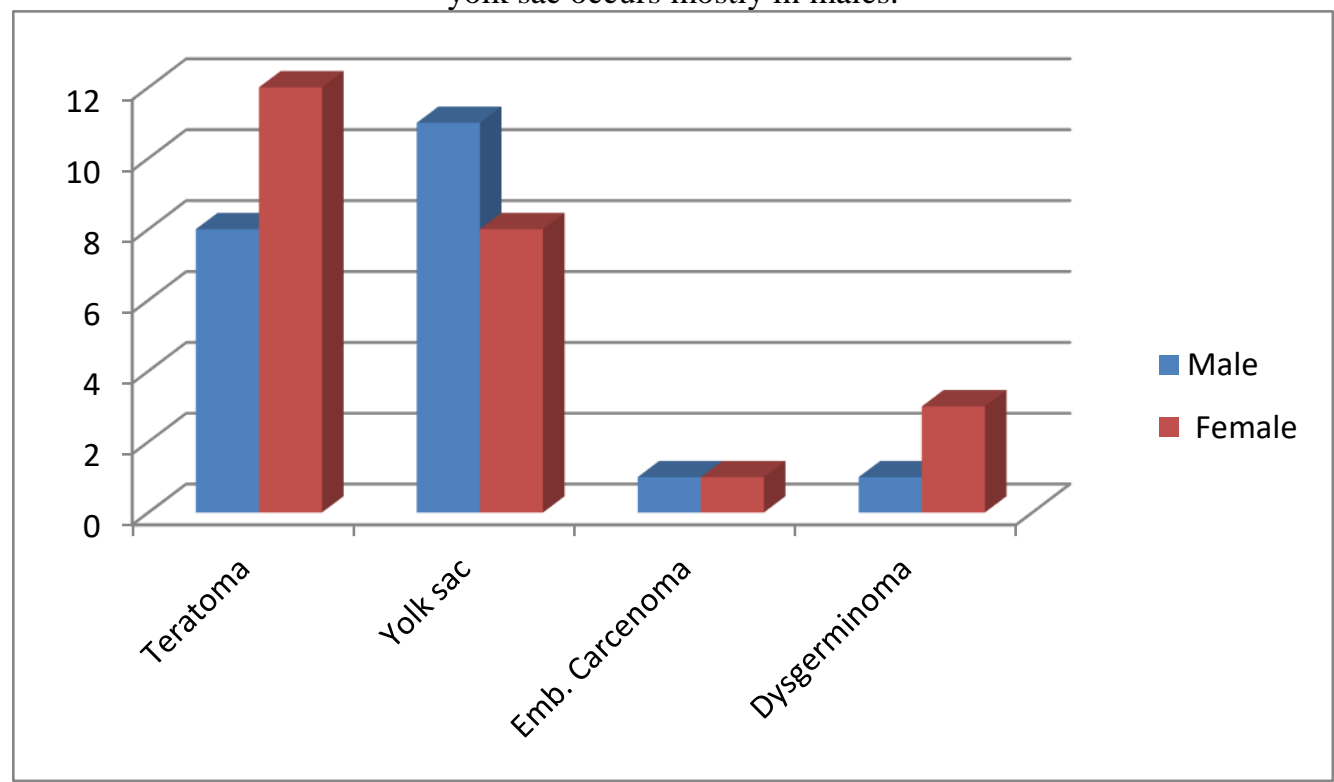

Fig 2. GCT histological distribution according to the sex.

The prevalence staging in GCT in the oncology Centre of Basra the most common was stage III 23 cases $(51.1 \%)$ follow by stage II was 10 cases $(22.2 \%)$ then stage I was 7 cases $(15.6 \%)$ and the last one was stage IV 5 cases (11.1\%). 
Table 8. Allocation of GCT according to the stagging system.

\begin{tabular}{ccc}
\hline Stage & No, & \% \\
\hline I & 7 & 15.6 \\
II & 10 & 22.2 \\
III & 23 & 51.1 \\
IV & 5 & 11.1 \\
\hline
\end{tabular}

Management of the GCT in all patients was surgical excision either for treatment or for biopsy followed by chemotherapy. Previously before the start, our collection of data in the oncology Centre of Basra used VAC (vincristine, actinomycin, cyclophosphamide) protocol .while in a period of our study used BEP protocol (cisplatin, bleomycin, and etoposide) as four to six courses depending on the stage of the disease.

Finally, the outcome of GCT in oncology Centre of Basra overall survival (OS) was $86.7 \%$, that during five years (60 months) follow up and the outcome Exitus six cases (13.3\%) two of them during treatment and the remainder four died during follow up, all of them were at a late stage (stage IV).

Table 9. Outcome of GCT in oncology Centre of Basra overall survival (OS)

\begin{tabular}{ccc}
\hline Outcome & No. & \% \\
\hline overall survival & 39 & 86.7 \\
Exitus & 6 & 13.3 \\
\hline
\end{tabular}

\section{Discussion}

Paediatric germ cell tumours (GCTs) are uncommon and various tumours are hypothesized to occur as a result of events in the uterus although that largely take place of unknown ethology[8]. GCT arises due to variation from normal differentiation of germ cells and includes a heterogeneous group of neoplasms with remarkable variability concerning histology and site of presentation: immature GCTs, malignant and Benign can be present in paediatric group with different rates, also according to age. The GCT histopathological types and sites differed with age and sex groups. The clinical characteristics found in this study are similar to other national and international studies related to the issue[9].

The most common age of occurrence of GCT was below 24 months 21 patients $(46.6 \%)$. second, the peak incidence of GCT occurs 1-23 months age group. This step of the result is like a study conducted by Özdemir, et al. Where the first peak was observed during the first 2 years of life. At less than one year old GCT was more incidence in females than males (M: F 1:2.5) there was a highly significant difference between the incidence of GCT in females than females in the first two years of life. While in 2-5 years old the female more involvement than male (F: M 1:1.1). The finding of this study agreed with Özdemir, et al., [2]. The teratoma was most patients 20 cases $(44.4 \%)$ mainly immature teratoma. Teratoma occurs highly incidence in females $(26.6 \%)$ and males $(17.7 \%)$ follow by yolk sac 19 cases $(42.2 \%)$ commonly occur in male patients 11 
cases $(24.4 \%)$ and female 8 patients(17.7\%). We excluded two patients who were diagnosed with mature teratoma and eliminated them in the evaluation of survival, staging, and follow regularly. Extragonadal site of origin was the most common (Sacrococcygeal and retroperitoneal regions) in 24 patients (53.3\%). the Sacrococcygeal was the most frequent extragonadal GCTs 20 cases(44.4\%), diagnosed during the first two years of life, half (50\%) occurred at the sacrococcygeal region.

The outcome in the oncology Centre of Basra has highly improved OS (86.6\%) as in Gobel U, et al. The prognosis in GCTs did not improve until the late 1970s when the VAC regimen became the standard chemotherapy in GCTs. Since the introduction of platinum-based agents(carboplatin and cisplatin) in paediatric chemotherapy protocol, the survival rates for children with malignant GCTs ranging from $75 \%$ to more than $90 \%$ [10]. In our follow up the OS was higher than another centre can explain by a little number of patients.

\section{Conclusion}

Our study demonstrates a correlation between tumour stage and survival. This can be explained by most Exitus patients associated with stage IV. The number of candidates in our series was little because our infrastructure capacity was very limited and depend on limited facilities. must be in the future used value of microRNAs as markers for enhancing the diagnosis and follow up the GCT and Elevated AFPand B-HCG level at the time of diagnosis and follow up had a beneficial effect on prognosis. In advanced stage and poor prognosis GCT advice used neoadjuvant (preoperative) chemotherapy to try to improve outcome.

\section{References}

[1] J. D. Fish, et al., Lanzkowsky's manual of pediatric hematology and oncology: academic press, 2016.

[2] S. İncesoy-Özdemir, et al., "Clinical and epidemiological characteristics of children with germ cell tumors: A single-center experience in a developing country," The Turkish journal of pediatrics, vol. 59, pp. 410-417, 2017.

[3] S. M. C. de Sousa Lopes, et al., "Proximal visceral endoderm and extraembryonic ectoderm regulate the formation of primordial germ cell precursors," BMC Developmental Biology, vol. 7, pp. 1-9, 2007.

[4] M. J. Murray, et al., "Identification of microRNAs from the miR-371 373 and miR-302 clusters as potential serum biomarkers of malignant germ cell tumors," American Journal of Clinical Pathology, vol. 135, pp. 119-125, 2011.

[5] L. Dehner, "Gonadal and extra gonadal germ cell neoplasms: teratomas in childhood," Pathology of neoplasia in children and adolescents, 1986.

[6] F. Shaikh, et al., "Paediatric extracranial germ-cell tumours," The Lancet Oncology, vol. 17, pp. e149-e162, 2016.

[7] I. Mahmood, "Prediction of drug clearance in children: impact of allometric exponents, body weight, and age," Therapeutic drug monitoring, vol. 29, pp. 271-278, 2007.

[8] J. N. Poynter, et al., "Trends in incidence and survival of pediatric and adolescent patients with germ cell tumors in the United States, 1975 to 2006," Cancer, vol. 116, pp. 4882-4891, 2010.

[9] M. Büyükpamukcu, et al., "Malignant sacrococcygeal germ cell tumors in children: A 30-year experience from a single institution," Tumori Journal, vol. 99, pp. 51-56, 2013.

[10] U. Göbel, et al., "Germ-cell tumors in childhood and adolescence," Annals of oncology, vol. 11, pp. 263-272, 2000. 